Results The results show that people who lived in the most deprived area were less likely to participate in arts activities (ATT $=-0.42,95 \% \mathrm{CI}=-0.55,-0.29, \mathrm{p}<0.001)$, attend cultural events $\quad(\mathrm{ATT}=-0.36,95 \% \mathrm{CI}=-0.46,-0.27, \mathrm{p}<0.001)$ or visit museums and heritage sites (ATT $=-0.39,95 \% \mathrm{CI}=-0.49,-0.29$, $\mathrm{p}<0.001)$. Sensitivity analyses testing on different sub-samples yield similar results. This indicates that our model successfully predicts low engagement in arts activities, cultural events and museums and heritage sites amongst those who lived in a deprived area across various populations.

Conclusion This study show that people who live in the most deprived area are less likely to participate in arts activities, attend cultural events or visit museums and heritage sites, independent of identified demographic and socioeconomic characteristics. Our findings suggest that addressing potential structural or neighbourhood factors may help increase individual motivation and capacity to engage in cultural activities.

\section{P63 THE SOCIAL AND PHYSICAL WORKPLACE ENVIRONMENT AND COMMUTE MODE: A NATURAL EXPERIMENTAL STUDY}

R Patterson*, D Ogilvie, J Panter. MRC Epidemiology Unit/CEDAR, University of Cambridge, Cambridge, UK

\subsection{6/jech-2020-SSMabstracts. 156}

Background Despite strong evidence for health benefits from active travel, levels in the UK remain low. Changes to the physical and social workplace environment might encourage active travel but there is little high quality evidence for this.

Methods Data come from 419 participants in the Commuting and Health in Cambridge study, who completed postal questionnaires in 2011 and 2012. Participants lived and worked in the Cambridge area and were predominantly recruited through their workplaces. Each participant's workplace environment was summarised using the number of physical characteristics from eight potential options (e.g. bicycle parking, shower facilities) and their level of agreement with five statements about workplace social norms around commuting (e.g. colleagues walk to work). We used a natural experimental approach to explore associations between changes in the physical and social workplace environment over time and changes in the proportion of commute trips i) exclusively by private motor vehicle, ii) exclusively by active modes and iii) including active modes, using fractional response logit regression in Stata 15, StataCorp. We additionally examined whether these associations differed between men and women.

Results In adjusted analyses, an increase of one physical characteristic was associated with a $2 \%$ (95\% confidence interval 0 to 4$)$ reduction in the proportion of commute trips by private motor vehicle and a $2 \%(95 \%$ CI 0 to 4$)$ increase in the proportion of commute trips which included active modes. In sex stratified analyses these associations were only seen in males, with a $3 \%(95 \%$ CI 1 to 6$)$ reduction in commute trips by private motor vehicle and an increase in commute trips including active travel of 5\% (95\% CI 3 to 8 ).

A change to more favourable social norms for walking or cycling among workplace management was associated with an increased percentage of commutes including active modes in women $(4 \%, 95 \%$ CI 1 to 7$)$ but not men. However, in both genders a change to more favourable social norms around colleagues' cycling was associated with reduced commuting by exclusively active modes $(-3 \%, 95 \%$ CI -5 to -1$)$.

Conclusion This study provides robust longitudinal evidence for sex differences in the associations between workplace environment and commute mode. Physical factors were associated with more active commuting in men, while the social environment appeared to have more complex associations that were stronger among women. Although this study was small and geographically circumscribed, its findings propound larger studies in more diverse contexts.

\section{P64 UNDERSTANDING PARADOXICALLY LOW RATES OF SELF-HARM IN A DEPRIVED, ETHNICALLY DIVERSE URBAN COMMUNITY IN THE UK: A THEMATIC ANALYSIS OF QUALITATIVE INTERVIEWS AND FOCUS GROUPS}

${ }^{1,2} \mathrm{C}$ Polling*, ${ }^{1} \mathrm{C}$ Woodhead, ${ }^{1} \mathrm{H}$ Harwood, ${ }^{1,2} \mathrm{M}$ Hotopf, ${ }^{1} \mathrm{SL}$ Hatch. ${ }^{1}$ Department of Psychological Medicine, Institute of Psychiatry, Psychology and Neuroscience, King's College London, London, UK; ${ }^{2}$ South London and Maudsley NHS Foundation Trust, London, UK

\subsection{6/jech-2020-SSMabstracts. 157}

Background Epidemiological studies show rates of self-harm through injury or poisoning vary substantially between different social contexts. Socio-economic deprivation predicts risk at individual and community level. However, despite high poverty rates, London has low rates of self-harm overall and contains highly deprived areas with paradoxically low rates. Using the stress process model as a theoretical framework, we explore why one such community exposed to multiple, chronic stressors, might nonetheless appear to have low rates of self-harm.

Methods This study forms part of a wider mixed methods project which used clinical data on service use following self-harm to calculate age standardised incidence rates by small-area in South East London, 2009-2016. These were combined with the Index of Multiple Deprivation to identify a case study area that was persistently deprived with below average self-harm rates. Semi-structured interviews were conducted with fourteen people working, paid or as volunteers, within community organisations serving the area. Two focus groups were conducted with 12 people currently resident in the area. Topic guides covered aspects of the area that impacted mental health positively and negatively, how people locally responded to distress and attitudes and responses to self-harm. A thematic analysis was conducted, with themes generated following discussion between two independent coders.

Results The case-study area was ethnically diverse, with a large Black population, which was reflected in the study sample. Participants reported that people in the community were exposed to multiple, chronic stressors related to marginalised social statuses and the social environment, with significant impacts on mental health. These were partly buffered by social resources related to community solidarity and an understanding of stressors as communal challenges, as well as a culture of self-reliance amongst individuals. However, identifying oneself as mentally ill, especially through being known to 